\title{
Agent-based Eight Wheel-legged Hybrid Wheelchair Control System
}

\author{
Dongxing Cao*, Shanshan Liu, Chao Wang and Minfei Li \\ Mechanical Engineering School, Hebei University of Technology, Tianjin, 300130, China \\ ${ }^{*}$ Corresponding author
}

\begin{abstract}
Eight wheel-legged hybrid wheelvhair robot motion is the process of multi-agent system collaborating work, in which each module can be viewed as an agent, such as frontal leg mechanism, latter leg mechanism, and seat position and orientation mechanism. Agent has the ability of sensing the environment, and at any time responds to the environment. The paper builds a new robot wheelchair. The user operates the handle to implement the communication of multi-agent, and changes the position and orientation of frontal leg, latter leg, and seat in the light sensing the environment. It can not only work on the ground, but also climb stairs and obstacles.
\end{abstract}

Keywords-multi-agent system; climbing wheelchair; robot mechanism; port-agent; control strategy

\section{INTRODUCTION}

Agent technologies are an effective method to realize intelligent control. Multi-agent collaboration can implement complexity system control. The wheelchair as a kind of transport instead of walk has been widely used by the elderly and the disabled. Campbell et al. ${ }^{[1]}$ presented a new design generation methodology called A-Design, which combines multi-agent systems, and automated design synthesis to realize electro-mechanical configuration design for changing user preferences. Miao et al. ${ }^{[2]}$ presented an intelligent-agent reasoning model, which has the ability to model, reason and make decisions on behalf of human beings. This model also has the ability to handle various types of fuzzy information. Rodrigo Antonio Marques et al. ${ }^{[3]}$ constructed a modular platform to facilitate the development of intelligent wheelchair through a multiagent system paradigm, at the same time, integrated all modules in the platform, and effectively reduced users' limitations, offering them a much more independent life.

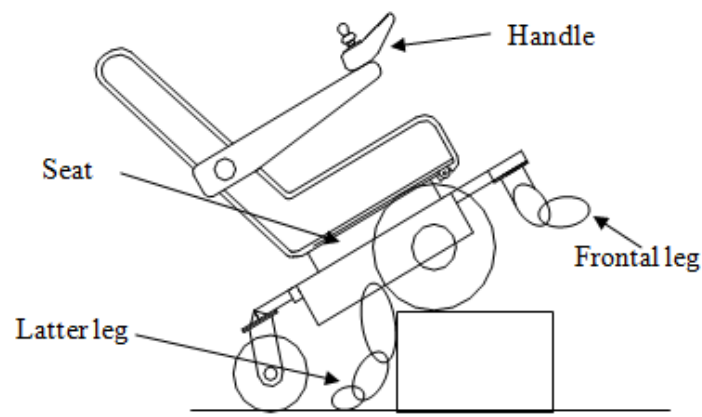

FIGURE I. THE ROBOT WHEELCHAIR CONFIGURATION

\section{EIGHT LEGGED HYBRID WHEELCHAIR}

\section{A. The Configuration of Wheelchair}

The configuration of eight wheel-legged hybrid wheelchair is shown in Fig. I, in which includes the frontal leg, latter leg, seat, and handle ${ }^{[4]}$. The frontal leg function is to realize climbing obstacle action when the wheelchair robot meets the obstacle. The latter leg function is to increase the drive force for climbing obstacle and adjust the direction of applying force. The seat function is to adjust the position and orientation of wheelchair robot gravity and keep the stability and safty of the rider. The handle function is to obtain the information of environment around obstacle and to control the wheelchair robot mechanism for completing various actions, such as climbing stairs, crossing obstacle, over the ditch, and so on.

\section{B. The Requirements of Control Strategy}

Agent technologies have been successfully applied to develop collaborative design ${ }^{[5]}$. In agent-based collaborative design system, agent has mostly been used for supporting collaboration among different mechanism blocks. It provides engineers an intelligent control strategy for robot motion that incorporates agent collaboration with an adaptive selection of paremeters. Similar to the A-Design system, port-agent system also provides a collaborative environment for the sharing of design information, data, and knowledge among distributed design team members ${ }^{[1]}$. In fact, much of the research work done in building agent-based collaborative design system has also focused on sharing information and data among agents. Unlike the webbased design system using the client/server architecture, a port-agent design system is a loosely coupled network of problem solvers that work together to solve problems that are beyond individual capabilities.

\section{AGENT-BASED FRAMEWORK}

\section{A. Multi-agent System for Wheelchair}

In order to realize an agent-based approach, it is necessary to build various function agents to collaborate them climbing stairs $^{[6]}$. A hybrid hierarchical agent architecture that is responsible for creating and collaborating different agents is proposed. It contains the Manager agent, Frontal leg agent, Latter_leg agent, Seat_agent, Communication_agent, Evaluation_agent, and so on. The transforming operations, the negotiation processes and protocols among the agents are described in detail. Agents in such system are communicative, 
collaborative, autonomous, reactive, and intelligent ${ }^{[5]}$.

\section{B. The Function of Multi-agents}

- Manager agent is used for controlling and coordinating all agents to solve the conflicts and exchange the message with the user. It includes conflict resolution agent and user interface $\operatorname{agent}^{[6]}$.

- Frontal leg agent is used for climbing stairs from sensing environment, and obtains command from Manager agent, at the same time, it can promptly keep two frontal leg at the same horizontal.

- Latter leg agent is used for adjusting applied force direction and position. Its act is controlled by the Manager agent, and receives command from the Manager agent.

- Seat agent is used for adjusting the gravity of wheelchair, and it can keep the seat at the horizontal position satisfied with the stability of the rider.

- Handle agent is used to control the wheelchair motion, especially it can seize the control rights of the Manager agent. In general, it works on the ground and climbing.
- Trunk agent is fixed as opposed to the other executing agents, it is one of the main perceptual system, and its trunk installs various sensors and moving mechanism, such as motors, clutch, and sensors, etc.

- Communication agent can provide the interactive function among agents by a standard syntax for message and use the knowledge query and manipulation language (KQML) for agent communications ${ }^{[7]}$. Here, it transmits the contents from data packet format with standard syntax, such as the paremeters of motors, sensors, clutch, and so on.

- Modification agent is used to revise and adjust frontal leg, latter leg, and seat to change or move the gravity and improve on wheelchair climbing action.

- Evaluation agent is used to evaluate the rationality of the paremeters in which a reasonable solution is adopted and bad solutions are deserted based on some stability criteria or rules for evaluation and behavioral models for decision making ${ }^{[4]}$.

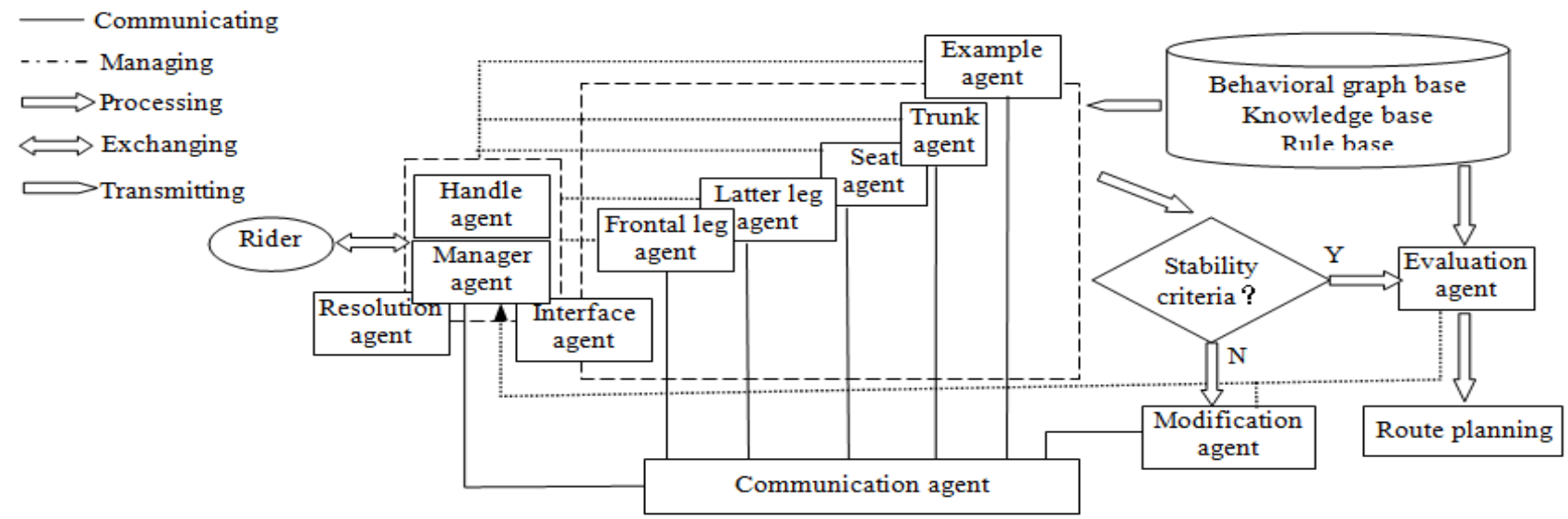

FIGURE II. THE FRAMEWORK OF MULTI-AGENT

\section{Control Strategies}

The agents are defined as behavior graph and knowledgebased strategies for solving control problems, they contain behavior graph, knowledge base, reasoning engine, and reasoning rules. In the current implementation, agents are not autonomous, but are triggered by system or by manager agents. The framework of agent-based intelligent control is shown in Fig. II. Different lines, arrow signs and graphs represent different indications, e.g. solid lines represent communication among agents and dotted lines represent manager agent may control and manage the other agents. These agents will constitute a federal-level alliance framework.

In the process of system work, the manager agent can control and coordinate other agents to solve the port behavioral model. At the same time, the evaluation agent may adopt some effective algorithm, such as fuzzy control, neural network, etc., to get optimal control paremeters. The system uses three main control methods during the inference process: forward-chaining inference (data driven), backward-chaining inference (goaldriven), and hybrid inference ${ }^{[8]}$. The forward inference phase is an obverse searching process while backward searching phase is a reverse searching process. If the result is a good solution, it will be put into the database as Exam-agent case. If there are no satisfactory solutions, the modification agent is fired by the manager agent and begins design solving again. In some cases, agents may attempt to adapt themselves to a better changing environment or goals. Agent adaptability includes what algorithm can be used to adapt the agent-solving, what search strategies can be used to adapt the agent-reasoning, how the maximum amount of changes can be foreseen in the future, how the system can be stopped in case it is out of control and how to deal with the adaptability which does not create the desired results. This framework has led to the implementation of a prototype system and valuable insights for wheelchair robot motion. 


\section{The Process of Multi Agent Work}

Each agent has an intelligent characteristics, and it can sense surrounding environment and respond to them. Multiagent work in coordination can implement a complexity control task of eight wheel legged hybrid wheelchair.

\section{A. The Description of Work Process}

Simple agent has an independent intelligent behavior. For example, frontal leg agent can sense stair obstacle and surroundings by the sensors, and it can adjust position and orientation of frontal leg by the aid of the effectors. Fig. III. represents the process of frontal leg agent work. At the same time, it receives the message from comm agent, and is controlled by manager agent. Similarly, latter leg agent, seat agent, and trunk agent are called executing agent that can sense surroundings and implement the actions.

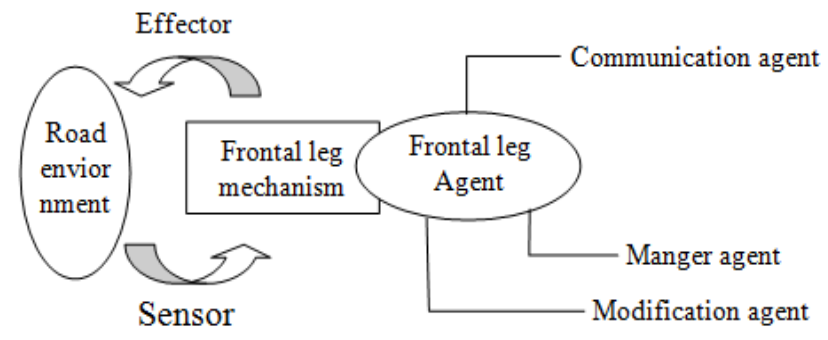

FIGURE III. FRONTAL LEG WORK

In the process of multi agent system work, Manager Agent can automatically control other agents to take actions. According to stability cone decision rules evaluation agent may get optimal control paremeters. In addition, handle agent can seize control by the rider decision. If the result is satisfactory, it will be put into knowledge base as example-agent case for later use. If not, modification agent is fired by manager agent and begins design-solving again. In some cases, agents may adjust the strategy and attempt to adapt themselves to better changing control paremeters or even control goals ${ }^{[5]}$.

When each executing agent obtains paremeters from sensors, they will be input fuzzy nerve net, and the hidden layer is used for resolving behavioral graph models, and transmits results to next behavior graph until output layer, and route planning is gotten as shown in Fig. IV.

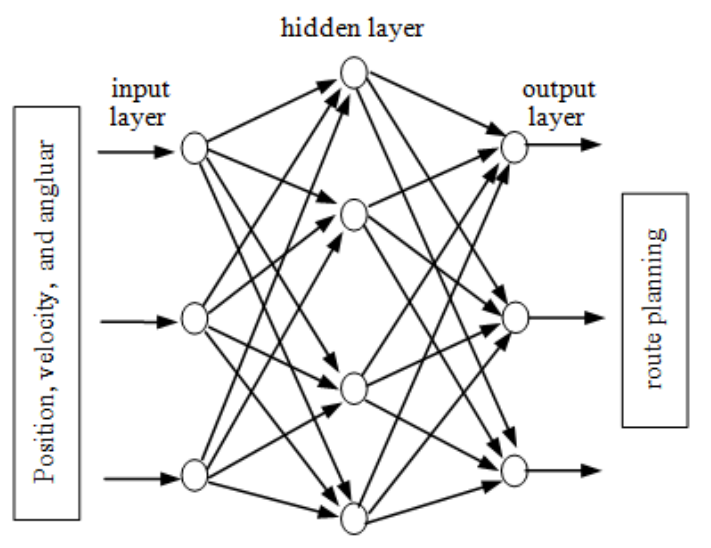

FIGURE IV. NERVE NET ALGORITHM
Conflict detection is a major concern in the process of system working, because some incidents, e.g. rule conflicts, storage capacity conflicts or technical conflicts, will happen at any time during the task execution. Therefore, the negotiation protocols should be used to resolve conflicts with the aid of the manager agent. For instance, stability priority and safety priority, etc. they form protocol rules for resolving conflicts and are put into the knowledge base ${ }^{[9]}$.

\section{B. Simulation Results and Analysis}

Consdering actual weight of wheelchair robot every parts we can obtain the total weight and the position and oraitation of the wheelchair gravity. Table 1 gives every paremeters correspond to each part in detail. And the number of displacement sensors, angluar sensors, and the clutches are given, and they can give the measuring results, such as displacement, volecity, and acceratication. These data can be input nerve net model and used for resolving behavioral graph models (see Fig. IV.). Thev control message can be obtained from nerve net algorithm, and used to control each excuting

TABLE I. WHEELCHAIR PAREMETERS

\begin{tabular}{|c|c|c|c|c|}
\hline Subtitle & $\begin{array}{c}\text { Mass } \\
\text { (kg) }\end{array}$ & $\begin{array}{c}\text { Num of } \\
\text { displacemen } \\
\text { t sensors }\end{array}$ & $\begin{array}{c}\text { Num of } \\
\text { angluar } \\
\text { sensors }\end{array}$ & $\begin{array}{c}\text { Num of } \\
\text { clutches }\end{array}$ \\
\hline Frontal leg & 15 & 4 & 2 & 8 \\
\hline Letter leg & 25 & 2 & 2 & 4 \\
\hline Seat & 90 & 1 & 3 & 1 \\
\hline Trunk & 25 & 4 & 6 & 2 \\
\hline Wheelchair $^{\mathrm{a}}$ & 155 & 11 & 13 & 15 \\
\hline
\end{tabular}

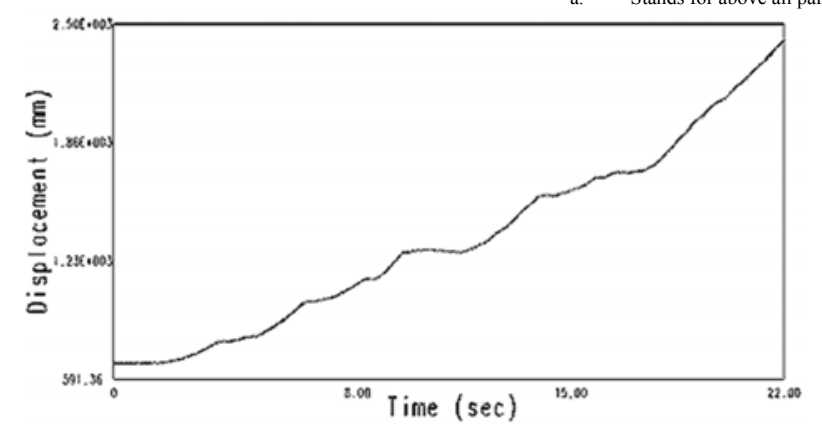

FIGURE V. THE DISPLACEMENT OF THE GRAVITY

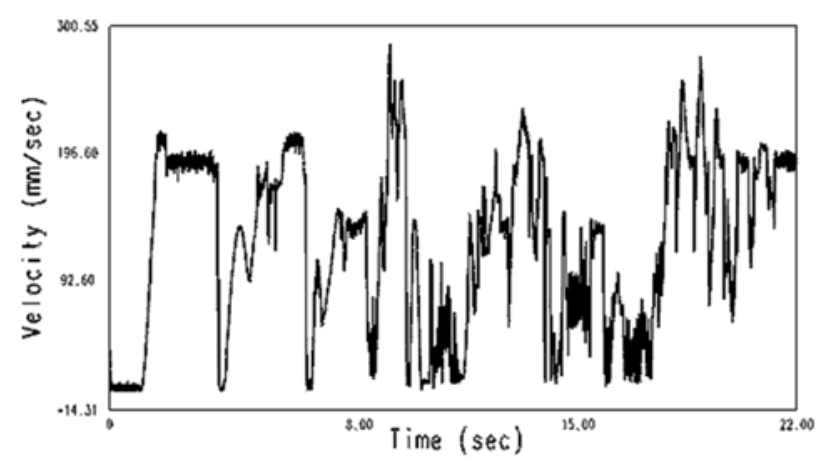

FIGURE VI. THE VELOCITY OF THE GRAVITY 


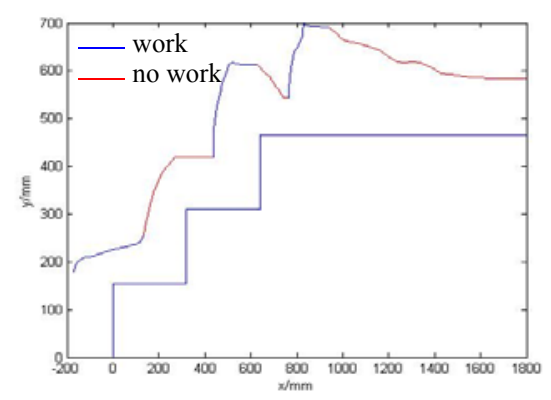

FIGURE VII. FRONTAL LEG CENTER TRAJECTORY

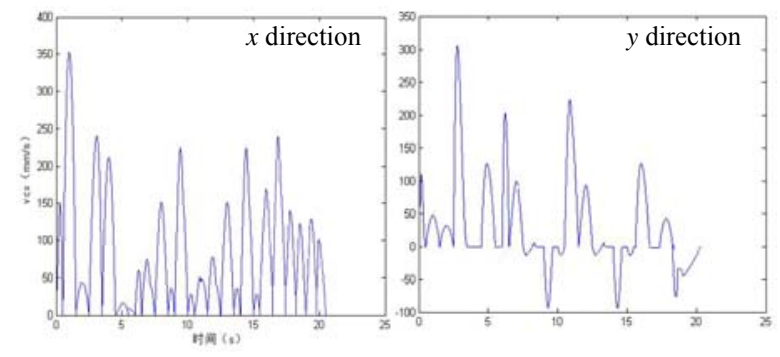

FIGURE VIII. FRONTAL LEG CENTER VELOCETY
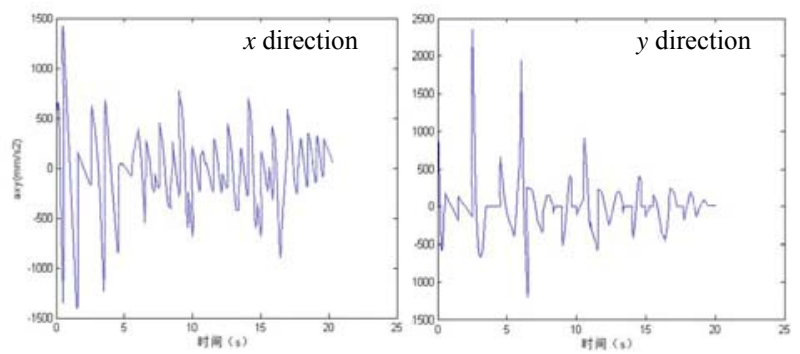

FIGURE IX. FRONTAL LEG CENTER ACCELERATION.

Agent act for climbing stairs. Fig. V. shows the gravity change relationship of wheelchair with time when climbing upstairs. The gravity velocity change affects the stability and safety of the rider, and its change range will be limited in a certain interval to keep the wheelchair stability shown in Fig. VI. It is one of the decisive factor for the foreleg act that the wheelchair can successfully climb up stairs. Fig. VII. shows the foreleg center trajection, in which blue line presents work state, and red line presents no work state. The correct trajection can keep the seat of the wheelchair horizontality and make the rider stable, at the same time, suitable velocity and acceleration will make the rider more safe and comfortable shown in Fig. VIII. and in Fig. IX.

\section{CONCLUSIONS}

The paper presents an agent-based strategy to eight wheelleg hybrid wheelchair robot control system. We have established a prototype system for limited application. Collaboration work is only used for the process of control strategy generation. We are extending the framework to include rider's intention as well, including to take back control role by handle agent. Dynamic transformation of the input to output layer of the nerve net model is being investigated. Current research is expanding this integration towards describing wheelchair robot system. We will further research agent-based approach to mechatronics control system, which provides the port knowledge to effectively present for multi domain problems.

\section{ACKNOWLEDGMENT}

The work was supported by NSF of China under Grant No. 51275152 and NSF of Hebei Province under Grant No. 2013202123 and Chinese Students and Scholars Studying abroad Funded Project of Hebei Province under Grant C201400306.

\section{REFERENCES}

[1] M. Campbell, J. Cagan, K. Kotovsky. Agent-based synthesis of electromechanical design configurations, Journal of Mechanical Design, 2000, 122: pp. 61 69.

[2] Miao, C.Y., Goh, A. and Miao, Y., Agent that models, reasons and makes decisions. Knowledge-Based System., 2002, 15, 203-211.

[3] B Rodrigo Antonio Marques, P Marcelo, R Luis Paulo, etc. Intellwheels: Modular development platform for intelligent wheelchairs. Journal of Rehabilitation Research and Development, 2011, 48(9): 1061-1076.

[4] Dongxing Cao, Zhanwei Li, Hui Li, Baolingzong Port-based ontology behavioral graph modeling for climbing robot route planning, WIT Transactions on Engineering Sciences, 2015, Vol.102: 117-123.

[5] G. Zhao, J. Deng, and W. Shen, CLOVER: an agent-based approach to systems interoperability in cooperative design systems, Computers in Industry, 2001, 45, 261-276.

[6] D.X. Cao, N.H. Zhu, C.X. Cui, et al. An agent-based approach to guiding the conceptual design of mechanical products, International Journal of Production Research, 2008, 46 (9): 2381-2396

[7] Finin, T., Peng, Y. and Labrou, Y., Mobile agents can benefit from standards efforts on interagent communication. IEEE Commun. Mag., 1998, 36, 50-56.

[8] Yanan Shan, Dongxing Cao, Longfei Lv, and Pan Qu Port-based ontology multi-scheme generation via genetic algorithm, Proceedings of the 10th International Conference on Frontiers of Design and Manufacturing, June $10 \sim 12,2012$, Chongqing, China

[9] Labrou, Y., Finin, T. and Peng, Y. Agent communication languages: the current landscape.IEEE Intell. Sys., 1999, 14, 45-52. 